\title{
Les formes compuestes «haber + participiu» n'asturianu oral: incidencia, funcionalidá y vixencia na fala cotidiana
}

por FÉlix Iglesias Fernández

\section{INTRODUCCIÓN}

$\mathrm{D}$ IENTRO'L MARCu de la filoloxía asturiana, l'enfoque tradicional del estudiu del sistema verbal considera esti constituyíu por formes verbales simples dafechu (ALLA, 200I: I78-I83), que conviven coles perífrasis iteratives tener + participiu, qu'espresen perspeutiva secundaria, tomaes como productu d'evoluciones patrimoniales de la llingua asturiana. Sicasí, ye un fechu sorrayable que na fala espontánea recuéyense casos de construcciones haber + participiu que puen indicanos la esistencia de compuestos coesistentes y permutables coles formes simples del verbu. Considérense un emprestu asimiláu dende la llingua castellana, llendáu sobre manera a les formes pluscuamperfeutes, futuru perfeutu ya infinitivu compuestu (ALLA 2002: 43; Bobes I96r: II6; Díaz Castañón 1967: 96), una incorporación seroña, ayena a la estructura intrallingüística asturiana (Martínez Álvarez 1973: 30I; García Arias et allii 1976: I19; Neira 1976: II9), favorecida por una situación de billingüismu socialmente desequilibráu (Andrés 1996: 37I). Con esto y con too, anque l'asturianu nun llegare a integrar dientro'l sistema verbal les 
formes compuestes colos valores propios que tienen estes nes llingües romániques que sí los xeneralizaron, nun se trataría tampoco d'un fenómenu exóxenu, porque hestóricamente sí esistieron formes compuestes del verbu rexistraes nel estadiu medieval del asturianu (Viejo Fernández 1998: 50-55).

L'enfotu d'esti trabayu ye afondar na incidencia, funcionalidá y vixencia de les construcciones compuestes haber + participiu nel asturianu contemporaneu y comprobar si los conteníos conseñaos respuenden a interferencies del castellán o, pela cueta, indiquen posibles desenrueldos intrallingüísticos del asturianu. De la mesma manera, comprobaráse la integración de les formes perifrástiques tener + participiu nel nucleu del sistema verbal asturianu y la so oposición frente a les formes simples del verbu.

Como la fixación de les formes haber + participiu ye desigual nel ámbitu asturianu, puesto que tienen mayor vitalidá nos sistemes centroorientales, nesta investigación escoyóse como oxetu d'estudiu la faza central asturiana.

\section{Presentación DE LOS DATOS}

\section{Metodoloxía ya informantes}

Como se vio hasta agora, los estudios del sistema verbal asturianu amuesen unos paradigmes complexos qu'intenten responder a la difícil cuestión sobre l'orixe y funciones d'unes formes compuestes consideraes estrínseques al asturianu. Tampoco nun ha escaecese, como reparen bien d'autores, ciertu enfotu por axustase a la llinia de la RAE n'aspeutos pragmáticos y semánticos estremaos pente'l sistema asturianu y el castellán; perclaru nel casu de los compuestos iterativos retrospectivos, tener + participiu del asturianu, colos tiempos compuestos haber + participiu en 
castellán, perfeutamente xeneralizaos y definíos por una clara oposición a les formes simples nesti últimu.

Persabiendo los datos anteriores, l'oxetivu que se propón esti trabayu ye:

- Comprobar la vitalidá y el rendimientu de los compuestos con haber + participiu y les perífrasis iteratives de participiu con tener nel asturianu central actual.

- Comprobar si los conteníos conseñaos respuenden a interferencies del castellán o posibles evoluciones intrallingüístiques del asturianu.

- Comprobar la integración de les formes perifrástiques tener + participiu nel nucleu del sistema verbal asturianu, oponiéndose a les formes verbales simples.

El métodu escoyíu pa esta investigación ye la entrevista oral, por ser la que conseña de manera más fidedigna y cenciella la fala, desaniciando asina les llimitaciones que pudieren aprucir d'un contestu de llingua escrita pa la so interpretación.

La manera más direuta, de mano, sedría facer una encuesta d'elicitación na que se-y pudiere entrugar al informante sobre l'usu que fai de les formes compuestes. Pero nun sedría tampoco la más afayadiza porque, anque'l ser humanu tea predispuestu de manera xenética al so adeprendizaxe, l'usu que se fai de la llingua ye un procesu automáticu ya inconsciente; cuando una persona fala nun se decata de lo complicaos que son los engranaxes llingüísticos nin los procedimientos mentales, polo que nun esiste la necesidá de planificar lo que va dicise, al contrario que nun ámbitu escritu. Poro, no tocantes al desendolcu mesmu de la entrevista, nun hebo tipu dalu de restricción nin obligación o direición nesi sen: permitióse-yos en tou momentu llibertá plena a les informantes entrevistaes pa que se desenvolvieren na fala xenuina de caldía. 
Dióse-yos prioridá a aquelles persones más vieyes del centru, centru-sur y centru-norte d'Asturies con ascendencia y nacencia asturiana. Esto implicó la necesidá de desplazase a los diferentes puntos xeográficos pa facilita-yos tolo posible l'actividá y evitar posibles situaciones d'estrañamientu qu'afectaren a la situación comunicativa o a la so salú.

Recuéyense nesti estudiu testimonios d'un total de nueve persones de puntos estremaos de la faza central asturiana. Estos sitios abarquen dende Güeñu (La Ribera), Uviéu, La Caizuela (Illas) y El Ferriru (Gozón). El númberu d'entrevistes alcordaes pa coles informantes diba ser mayor nun principiu, pero por motivos ayenos a la mio voluntá, el númberu final de les entrevistaes resultare inferior al previstu en primeres; al tratase de falantes d'edá avanzada, nesti tiempu munches d'elles vieran mermaes les sos capacidaes cognitives y otres finaren.

Nun se van desendolcar equí les carauterístiques propies de caúna de les variedaes diatópiques de la llingua asturiana, pero sí ye d'interés mentar obres de referencia nesti sen: I) El bable del Cabo Peñas: Contribución al estudio del bable central, Díaz Castañón (1966); 2) Aspectos del bable occidental, de Rodríguez-Castellano (1954); y 3) Las lenguas circunvecinas del castellano: cuestiones de dialectología hispano-románica, de Catalán Menéndez-Pidal.

Los y les informantes que se recueyen nel estudiu son los que van darréu:

I. Elena González Fernández (Lena), 8o años, ye nacida en Grameo (Santa Cruz, Mieres), onde pasó los primeros años de la so vida. Pasó la so mocedá pente Quintaniella y Güeñu hasta qu'al rodiu los venti años movió pa Uviéu pa trabayar d'empleada nel hotel La Reconquista hasta la so xubilación. Reside anguaño n’Uviéu.

2. Francisca Alonso Fernández (Paqui), prima d'Elena, 78 años, ye nacida en Llatores, anque pronto la familia se mudara a Quintaniella. Residiera ellí brevemente hasta que, darréu, marcharon pa La Manxoya y, finalmente, pa Uviéu, onde pasó la mayor parte de la so vida hasta anguaño. Trabayó en voluntariáu cuidando neños. 
3. Benito Álvarez Cárcaba (Benitín), 74 años, ye nacíu en Güeñu, onde pasó la mayoría la so vida, xunto con Uviéu. Trabayó na central térmica de Ferreros hasta que se xubiló y volvió pa Güeñu, onde reside anguaño.

4. Marcelina Rotella de Álvarez, muyer de Benito, ye nacida Riosa, 73 años. Movió pa Güeñu cuando casó de moza y vivió tamién n’Uviéu hasta que volvió finalmente xunto con Benito. Dambos residen en Güeñu anguaño.

5. Raúl López González, 63 años, ye nacíu en Güeñu, onde residiera tola vida de manera ininterrumpida, con desplazamientos llaborales pa Uviéu. Trabayó nel sector mecánicu, chapista, y na construcción hasta la so xubilación. Anguaño reside en Güeñu.

6. Rosa García (Rosina’l Campu), 96 años, nacida n’El Ferriru (Gozón). Trabayó cómo criada n'Avilés y Xixón hasta que casó de moza y tornó pa El Ferriru, momentu nel que se dedicó a trabayar na casería. Tola so vida se desendolcó nesi pueblu nun siendo a ralos, pente los doce y los dieciséis años y los diecisiete y los venti.

7. Margarita González Ovies (Margarita la Xenra), 82 años, ye nacida n’El Ferriru (Gozón). Tola so vida la desendolcare n’El Ferriru y nel trabayu de la llabranza.

8. Bienvenido Carlos González Ovies (Venido la Xenra), 77 años, ye nacíu n’El Ferriru (Gozón). Vivió siempre n’El Ferriru, anque tuvo trabayos nos astilleros d'Avilés, calteniendo siempre'l so domiciliu y desplazándose caldía pa trabayar. Depués cambió esos trabayos pola casería, quedando definitivamente n’El Ferriru.

9. Jose Manuel Reguero (El Maestru), tien 66 años y ye nacíu n'Illas. Foi maestru y direutor d'escuela hasta la so xubilación, alternando tamién cola so afición d'actor de teatru. Anguaño reside en La Caizuela, pero tamién tuvo, por motivos llaborales, en Cabanaquinta, n'Avilés y n’Uviéu, onde estudió na Escuela Universitaria de Maxisteriu.

Otra manera, estos informantes repartiéronse en grupos siempre que foi posible pa entamar una charra dinámica que permitiere la interaición informal pente ellos de manera bonal.

Atopamos los siguientes grupos:

N’Uviéu:

Io. Elena González Fernández

II. Francisca Alonso Fernández 
Tamién s’entrevistare a Elena González Fernández de manera independiente.

En Güeñu:

I2. Benito Álvarez Cárcaba

I3. Marcelina Rotella de Álvarez

I4. Raúl López González

I5. Elena González Fernández

Tamién s'entrevistare a Elena González Fernández y Raúl López González como otru grupu aparte.

N'El Ferriru:

I6. Rosa García García

17. Margarita González Ovies

I8. Bienvenido Carlos González Ovies

N'Illas:

19. Jose Manuel Guerrero

En resultancia, les grabaciones sumen un total de más de diez hores de conversación. Trescríbense namás les muestres contestuales onde apaecen exemplos de construcciones compuestes colos verbos haber, infinitivu compuestu con haber y tener.

\section{Delles observaciones sobre'l casu de Güeñu}

En Güeñu nun se conseñó nenguna forma compuesta coles construcciones haber + participiu nin tampoco coles perífrasis de perspectiva secundaria tener + participiu na fala espontánea de los informantes; los únicos compuestos qu’apaecieron mentólos Benito Álvarez Cárcaba -y Marcelina Rotella de Álvarez pa sofitar puntualmente lo dicho por 
aquel- y fíxéralo como resultáu de la esplicación sobre la temática de la investigación que yo-yos daba a tolos informantes. Poro, nun se pue dicir que se trate de muestres de la fala espontánea porque’l so usu nun aprució bonalmente sinón condicionáu pola mio esplicación. Amás, el falante mentáu fizo un usu puntual de les formes que yo utilizara nel intre, pero non como parte de la so fala, sinón como exemplificaciones pa intentar aportar una rempuesta especulativa de les diferencies coles formes verbales d'acordies cola so perceición. Sicasí, en nengún otru momentu de la charra xurdió forma compuesta na fala espontánea. Sí se conseñen formes verbales simples de pretéritu pluscuamperfeutu d'indicativu ya imperfeutu de suxuntivu. Por exemplu, al esplicar el casu concretu espuestu por Martínez Álvarez (1973: 305-306) sobre la diferencia pente matáronlu cuando la guerra, matárenlu cuando la guerra y hubiérenlu matao cuando la guerra, onde'l conteníu d'anterioridá na forma compuesta ye mayor que respeuto a la forma simple:

20. Benito - En bable, no sé, pero nosotros decimos murió cuando la guerra. Muriera, no; yá queda un poquitín n'interrelación porque yá ye pasáu. Hubiere muerto, ye casi preguntando; si dices hubiere muerto no afirmes que murió.

2I. Marcelina - Hubiera muerto, ye una duda.

22. B - Por ejemplu, dices: El maríu de Lena pasólo mui mal durante la guerra; ¡hubiere muerto! Eso d'hubiere muerto ye porque pudo morir o no. Tu nunca afirmes namás que si dices murió. N'asturiano, eh. O si dices: l'otru día tuve un problema col coche, ostie, mira que si me sal un poco mal, hubiere muerto. Pero toi equí.

Con estes, tienen abondu interés morfolóxicu poles importantes diferencies pa coles del estudiu de Josefina Martínez Álvarez (I973). Si nesti s'observen valores temporales d'anterioridá n'indicativu na forma compuesta hubiera matao, en Güeñu la mesma entiéndese con valor modal y non terminativu. 


\section{ANÁLISIS}

\section{Resultaos estadísticos}

Conséñense nesti estudiu les siguientes estadístiques:

N'Uviéu, 8 formes compuestes con haber + participiu: hubiese valío; hubiesen llevao; hubiesen pasao; Hubiese echao; Habien tapao [x2] y hubiesen tapao; y Hubiere + participiu [incompleta]. 4 formes compuestes d'infinitivu con haber + participiu: haber quemáolu; haber pisáolu; haber enterráolu; y haber preguntáo-y.

No tocantes a les perífrasis iteratives retrospectives de tener + participiu, apaecen 4 exemplos: tien traíote y téngote sacao; tiénmelo dicho; y No lu tengo tratao.

N'El Ferriru nun s'alcontraron usos que recoyeren formes compuestes cola construcción haber + participiu nin tampoco cola construcción d'infinitivu compuestu con haber. Por embargu, conséñense construcciones perifrástiques de participiu: tiénenme buscao y tengo oío.

N'Illas alcuéntrase una forma qu'aparentemente sedría una d'infinitivu compuestu con haber + participiu (de no haber... [incompleta]). Ye probable que se tratare d'un participiu anque nesa mesma oración l'informante la dexare en suspenso.

En Güeñu apaez la forma hubiere + participiu coles carauterístiques comentaes.

La forma mayoritaria que vemos nes muestres ye la construcción cola forma hubiese + participiu, conseñada 5 vegaes, a la que-y hai que sumar la equivalente hubiere + participiu, qu'apaez 2 vegaes. En xunto, 8 .

Enantes d'entrar a analizar los datos pormenorizadamente, podemos sacar unes primeres conclusiones en base a ellos porque, lloñe d'espeyar resultaos inesperaos, estos datos sofiten lo previsto nos estudios de la 
materia. Primero, que l'emplegu más notable d'una forma compuesta con haber conxugáu apruza na franxa del pluscuamperfeutu, tanto cola forma habia + participiu como con hubiera,-e / hubiese. Esta última ye la qu’amuesa una vitalidá enforma mayor y prácticamente esclusiva na fala. Segundo, que se conseñen tolos compuestos con haber + participiu nuna zona urbana como ye Uviéu ya, inclusive, que nes zones rurales pueda nun apaecer nin siquiera l'infinitivu compuestu. Y, a últimes, respuende tamién a les previsiones que suceda a la inversa cola forma tener + participiu. La perífrasis iterativa ta presente nos dos ámbitos más estremaos, l'urbanu uvieín y el rural gozoniegu, calteniendo les semeyances a nivel formal cola inmovilización en neutru de los participios.

\section{I. Exemplos haber + participiu}

I. Si no la ficiera nun me hubiese valio pa ná.

2. Lloca al saber que lu hubiesen llevao elles.

3. Coses de que hubiese pasao cuando ella se criaba.

4. Si lo hubiese echao... comiólo... echólo según lo comió.

5. Lo qu'habien tapao.

6. La nariz qu'bubiesen tapao.

7. Nunca supe yo qu'Ángeles hubiere... fuere a coser a casa Otilia.

L'exemplu I ye una construcción condicional col verbu espresáu en mou suxuntivu. Anque ye más frecuente alcontrar la forma compuesta na prótasis, equí vemos un casu nel que s'alluga na apódosis, mentanto que’l pretéritu imperfeutu de suxuntivu ficiera allúgase nel primer términu ya introduznos nel supuestu hipotéticu. Nun habría cambéu dalu nos conteníos de la oración condicional si cambiáremos la forma compuesta pola forma equivalente. Por exemplu: si nun la ficiera nun me hubiese valio pa ná = si nun la ficiera nun me valiera pa ná. 
El xeitu peracabáu de l'aición pasada, ensin influencia nenguna nel presente, recuéyese cola forma simple del pretéritu pluscuamperfeutu d'indicativu n'asturianu n'oraciones condicionales. Nesti contestu (condicionales irreales) anúlase la diferencia modal col pretéritu pluscuamperfeutu de suxuntivu castellán (bubiese + participiu) y nun s'aprecia nengún aporte relevante aspeutual, temporal nin modal.

Hubiese valio presenta un valor potencial equivalente a los compuestos castellanos de condicional perfeutu d'indicativu (habría valido) y pretéritu pluscuamperfeutu de suxuntivu (hubiera o hubiese valido). Esta rellación d'equivalencia en construcciones qu'amuesen una probabilidá futura nel pasáu dase tamién n’asturianu ente’l pretéritu pretéritu imperfeutu de suxuntivu (la forma indicativa del pluscuamperfeutu) y condicional del mou potencial: si nun la ficiera nun me valdría pa nada = si nun la ficiera nun me valiera pa nada).

La torna al castellán normativu aceutaría l'usu del pretéritu pluscuamperfeutu de suxuntivu tanto en prótasis como n'apódosis, pero tamién esiste la opción d'intercambialu por un condicional perfeutu (habría + participiu) na segunda cláusula. Al neutralizase les distinciones modales tendríemos: si no la hubiera hecho no me hubiera valido para nada; o con condicional, si no la hubiera hecho no me habría valido para nada.

Ye llamativo que la oración tenga una significación que se sal de l'argumentación sostenida pola informante sobre la inutilidá de facer la comunión: afirma que lo único que recibiere foi una pamelada (posible alteración de palmada o palmetada o aceición negativa del sombreru pamela) y, darréu, que nunca nun-y la pidieran pa nada. Esta anomalía podría debese a una confusión propiciada por una integración errática de los compuestos d'orixe castellán. Por embargu, tamién ye posible que se deba a un error percomún atribuyible a contestos de fala oral ya in- 
formal; anque tengan una posición sintáutica atípica, el valor semánticu nun dexa de ser el mesmu.

Pa siguir coles construcciones condicionales, nel exemplu 4 tenemos una construcción modalmente marcada (condicional) asemeyada a la vista enriba. Esta vegada la forma compuesta apaez na prótasis ( $\mathrm{Si}$ lo hubiese echao...). Tamién tien problemes d'interpretación: nun hai apódosis espresada, yá que la informante interrumpiere la elocución y dexárela inconclusa. Podría hipotetizase sobre'l posible usu d'una forma compuesta hubiera + participiu, como na anterior, o un cambéu d'orde implicando una forma verbal non compuesta. En tou casu, esiste la necesidá d'una coordinación de dos formes verbales si consideramos que se trata d'una oración condicional irreal.

Centrándonos agora no que sí figura, la situación que s’espeya ye de desaniciu de la forma de pretéritu pluscuamperfeutu asturianu (ficiera) en cuenta del compuestu hubiese echao. Si alternamos cola forma simple, tendríemos si lo echare, que podríemos inxertar nuna oración completa de condicional, por exemplu: si lo echare fuera de l'alfombra, nun me daba / daría / diera más; o si lo echare en cachinos nun me daba / daría I diera más.

Coles mesmes, col compuestu infierse una intensificación nel matiz terminativo-resultativu de tirar, de manera análoga a la que menta Martínez Álvarez (1973: 306-307) y recueye Viejo Fernández (I998: 45) n'hubiera + participiu pa funciones d'indicativu. Ye más difícil percibila na forma simple.

Otra posible interpretación ye considerala una oración simple desiderativa (;Si lo hubiese echao...[fuera]!), qu'apuerta un refuerzu espresivu modal, coherente coles situaciones d'apaición de los compuestos.

Nos exemplos 2 y 3 tenemos otros casos cola forma compuesta hubiese + participiu. Esta forma recueye'l conteníu del pretéritu pluscuamperfeutu d'indicativu del sistema castellán (Habian lleva[d]o) que, al 
mesmu tiempu, tien el del pretéritu pluscuamperfeutu d'indicativu simple- asturianu (llevaren). Dambes faen referencia a fechos allugaos nun momentu anterior al que se toma como referencia: llevar el gatu y vivencies de cuando se criaba. Al dase esa situación nos dos exemplos, puen sustituyise poles correspondientes simples (Martínez Álvarez 1973: 303-307; Cano González 1976: 54-55; Viejo Fernández 1998: 45-48), resultando: Lloca de saber que lu llevaren elles; y Coses [de] que pasare cuando ella se criaba. Obsérvase la neutralización modal indicativu-suxuntivu de manera clara nel complexu hubiese + participiu n'asturianu. Esta forma, en principiu, ye la del pretéritu pluscuamperfeutu castellán de suxuntivu, pero l'usu de les informantes converxe nuna función d'indicativu que-y ye propia a la forma simple asturiana. En xeneral, nun s'albidra diferencia significativa ente'l tiempu verbal qu'espresa la forma compuesta en términos resultativos o terminativos frente a la forma simple.

Tanto l'exemplu 2 como 3 danse en contestos sintáuticos que marquen gramaticalmente la fonte d'información de la enunciación. Vese espresamente nel exemplu 2, onde se sorraya más al introducise'l compuestu tres d'un verbu parentéticu: lloca al saber que lu hubiesen llevao elles. Nel exemplu 3, la informante teoriza sobre les probabilidaes de que la persona aludida tenga dalguna alcordanza de Güeñu, yá que mudó de mui neña pa Uviéu y considera qu'estes son mínimes. Nun apaez de manera esplícita un verbu parentéticu, como nel exemplu 2, pero ye vinculable a les carauterístiques de la llingua oral ya inferible pel contestu nel qu'asocedió.

Los exemplos 5 y 6 guarden rellación al apaecer siguíes nel momentu de la charra coles informantes Francisca Alonso Fernández y Elena González Fernández -el grupu d'Uviéu- respeutivamente.

De mano, esti exemplu ye la comprobación esplícita de lo conseñao nos exemplos anteriores sobre la neutralización modal indicativusuxuntivu nos pluscuamperfeutos n'asturianu: pue vese nes subordinaes 
axetives lo qu'habien tapao y la nariz qu'bubiesen tapao que los valores de los complexos verbales ehí espresaos anulen les sos oposiciones modales en favor de la d'indicativu. Al empar, ye la variante hubiese + participiu la que s'impón y la qu'asume esti valor.

La forma habia + participiu ye otra forma compuesta d'incidencia na fala (Neira 1976: II9) con distribución xeográfica fundamentalmente nos núcleos urbanos; ye'l casu d'Uviéu, lo que pue evidenciar un refuerzu d'esa forma por presión del sistema castellán. Darréu d'ello, nun implica que seya propiamente castellana, yá que tien dellos usos que s'aprecien de calter llocal y autóctonu en sitios concretos (Viejo Fernández I998: 40-45), sobre manera al dase la situación de neutralización con hubiera,$e$ / hubiese + participiu, que ye una forma que pue presentar valores indicativos y suxuntivos. Sicasí, lo que se conseña equí ye probable que respuenda a un calcu del castellán.

L'exemplu 7 ye una oración subordinada sustantiva en función de commplementu direutu (CD) onde la forma verbal del pretéritu pluscuamperfeutu d'indicativu fuere apaez col compuestu hubiere + participiu; facemos esa suposición porque, de nun interrumpila, con toa probabilidá y por inferencia contestual la informante utilizaríala con un participiu (dío). Por embargu, lo que finalmente figura na grabación ye cómo s'interrumpe y rectifica aquel compuestu pola forma verbal pluscuamperfeuta asturiana nun contestu retrospectivu inactual. Nun fai falta facer cambéu dalu porque paez apreciase que la falante decántase pol fuere en cuenta del hubiere [dío] al tomar la primera como una forma asturiana, equivalente pa espresar el conteníu.

D’otramiente, el compuestu vuelve a apaecer, como nos exemplos 2 y 3, nun enunciáu en rellación de subordinación introducíu por un verbu parentéticu qu'implica cognición (Nunca supe yo qu'Ángeles hubiere... fuere a coser a casa Otilia) nun contestu evidencial. Poro, reforzando'l calter probabilísticu de la construcción. Tamién podría 
deducise, pola la reformulación instantánea del nucleu oracional de la subordinada, que los compuestos haber + participiu constituyen un importante marcador llingüísticu qu'afita una llende autoconsciente ente asturianu y castellán.

Nesti puntu puen vese dellos patrones reincidentes na casuística de los complexos verbales con haber. A esceición de los exemplos i y 4 -condicionales-, tolos demás son oraciones subordinaes en contestos evidenciales, indicativos de contestos psicolóxicos, introducíos por verbos parentéticos de manera esplícita (exemplos 2, 3 y7) ya implícitos contestualmente (exemplos 5 y 6 ) nos qu'una informante actúa como fonte d'información pa otra. Amás, de los exemplos 5 y 6 conséñase de manera manifiesta la neutralización modal na adautación indicativusuxuntivu na esfera de los pluscuamperfeutos.

\subsection{Los exemplos de Güeñu}

Aclarao lo especial del contestu nel que xurdieron, podemos facer les siguientes apreciaciones sobre los dos exemplos a los que recurriera l'informante pa intentar esplicar les sos hipótesis. Los exemplos son:

I. El maríu de Lena pasólo mui mal durante la guerra; ;hubiere muerto!

2. L'otru día tuve un problema col coche, ostie, jmira que si me sal un poco mal hubiere muerto!

Equí alcontramos dos oraciones yustapuestes. El valor que l'informante afirma pa esta forma compuesta ye retrospectivo-probabilísti$\mathrm{cu}$, porque pudo morrer o non; l'aición de morrer pudo asoceder de cumplise determinaes circunstancies que nun se dieron pero fueron posibles. Esto fai que se desanicie dafechu la posibilidá de que faiga referencia a una forma de futuru perfeutu de suxuntivu en castellán normativu (bubiere + participiu), yá en desusu na fala oral. Poro, aseméyase más a una utilización pluscuamperfeuta correspondiente cola forma castellana 
del suxuntivu (hubiera o hubiese + participiu) o condicional perfeutu (babria + participiu).

Nel casu de tratase d'una construcción condicional, el falante castellán podría sentir esti usu como incompletu, a la escontra del asturianu -faciendo difícil xustificalo como castellanismu- y echar en falta la construcción protática (habría muerto si...) pa completar la condicional hipotético-probabilística que tendría consecuencies nel presente (morrer). Tamién ha desaniciase que se trate d'un usu pluscuamperfeutu, porque xeneraríase otra confusión al entender habría muerto o hubiera, - $e$ pola so carga desiderativa en castellán: El marido de Lena lo pasó muy mal durante la guerra. ¡Le valdría más haberse muerto / pudo haber muerto! Nun ye esta la llectura que s'obtién del contestu probabilísticu de la forma compuesta ¡hubiere muerto!, cercanu al de la perífrasis hubi morrer, qu'espresa la proximidá de que daqué va asoceder nun momentu percecanu: El marín de Lena pasólo mui mal durante la guerra, ¡hubo morrer!

$H u b i$ + infinitivu consénase como un usu de les formes perifrástiques d'infinitivu con haber (dela) + infinitivu, qu'espresa obligación. Dientro d'esta categorización, paez factible que s'espeye tamién con una perífrasis modal d'infinitivu compuestu d'haber + participiu, marcadamente probabilística, y un verbu auxiliar hipotéticu o desiderativu: El maríu de Lena pasólo mui mal durante la guerra; ;podía haber muerto! Paez nidio que lo que se diz ye pa reforzar la mala situación del maríu de Lena, anque nun tien que s'inferir necesariamente que morriera, sinón qu'esistió probabilidá enforma de qu'aquel pudiere morrer cuando la guerra - perspeutiva retrospeutiva inactual.

Podia + haber (n'infinitivu) + participiu recueye tanto valores desiderativos como probabilísticos; esti últimu ye al que se fai referencia. Esta perífrasis modal pue tener un desendolcu patrimonial cuando apaez en determinaos contestos con poder y tener d'auxiliares en forma personal inactual (Viejo Fernández 1998: 52-53). Esto propiciaría un usu difícil- 
mente catalogable como castellanismu, porque s'alloña de cualquier usu d'esti complexu verbal naquel sistema llingüísticu.

L’exemplu 2 de Güeñu tamién podría aplicase'l mesmu procesu: ¡Si me sal un poco mal podia haber muerto!, en cuenta de hubiere muerto. Anque nun ye esautamente'l mesmu casu porque tamos énte una construcción condicional con prótasis y apódosis (si me sal un poco mal + hubiere muerto) na que'l primer verbu ye un presente d'indicativu asturianu y el segundu un compuestu hubiere -alternante con hubiera-acompañáu de participiu.

Equí, esta construcción d'infinitivu compuestu con haber + participiu nun se correspuende de manera esauta cola forma verbal simple podía morrer: Si me sal un poco mal podía morrer escarez de la carga aspeutual perfeutiva que sí s'infier de l'aición hipotético-probabilística de morrer cola compuesta si me sal un poco mal podía haber muerto. Ye una condicional irreal construida como realizable, anque trate una aición hipotética inautual, porque l'informante considérala psicolóxicamente próxima.

Ye perinteresante que los informantes incidan nel valor probabilísticu -modal- per aciu de la forma hubiera + participiu en contestos que s'identifiquen col infinitivu compuestu d'haber. Y sobre manera conscientemente y basándose namás na so esperiencia. Utilizóla como exemplu porque foi'l que se punxo como modelu esplicativu pa esponer l'oxetivu de la investigación. Pero, a diferencia de los matices terminativos d'anterioridá recoyidos por Martínez Álvarez (1973: 306) en San Cloyo, en Güeñu los informantes concibiéronla con un conteníu modal y non terminativu, en vez de col matiz temporal d'anterioridá, que ye lo más sorrayao na forma compuesta. Estos conteníos probabilísticos son los qu'en dalgunes perífrasis modales d'infinitivu compuestu podríen tener un desendolcu funcional autóctonu del asturianu (Viejo Fernández I998: 54). 
Enllazao direutamente colo anterior, les construcciones d'infinitivu compuestu con haber + participiu, anque seyan perífrasis perfeutamente normatives, son les menos abondoses de les muestres: conséñense 4 exemplos ente los qu'unu d'ellos, el cuartu, forma parte d'una oración truncada.

\subsection{Exemplos d'infinitivu compuestu}
I. Tenia qu'haber quemáolu.
2. Debiin d'haber pisáolu.
3. Tenia qu'haber preguntáo-y a Amparo.
4. Eso ye culpa mía de no haber... [estudiao]
5. Tenien qu'haber enterráola.

Nos exemplos I, 2, 3 y 5 tenemos construcciones del denomáu infinitivu compuestu con haber + participiu. Trés d'elles (exemplu I, 3 y 5 ) auxiliaes por tener conxugáu en pretéritu imperfeutu d'indicativu (tenía que + haber [n'infinitivu] + participiu). Nelles, tenía qu'haber quemáolu, tenía qu'haber preguntáo-y a Amparo y tenien qu'haber enterráola atopamos dos interpretaciones:

a. Deséu non realizáu o conveniencia de quemar el regalu que-y fixera nun pasáu inactual a una persona desagradecida / face-y la pregunta a Amparo / qu'enterraren a la so maestra sobre'l suxetu naquel marcu temporal concretu; y

b. Obligación non realizada que tenía de quemalu / de pregunta-y a Amparo sobre la identidá d'aquel suxetu / de qu'enterraren a la maestra pa que nun-y pegare na escuela.

La informante da-y un sentíu retrospeutivu; poro, falamos del casu A. Si intercambiamos el compuestu (haber quemáolu / haber preguntáo-y / haber enterráola) poles correspondientes formes simples (tenía que quemalu / tenía que pregunta-y / tenien qu'enterrala) vemos que, nel 
mesmu marcu modal, puede llegar a marcase morfolóxicamente'l valor d'anterioridá terminativa.

El sen precisu que la informante da a la oración nun paez posible alternando la forma compuesta cola simple, sofitando lo qu'afirma Viejo Fernández (1998: 53) sobre la oposición funcional nestos complexos. Al tratase de formes inactuales, tampoco nun se pue algamar el mesmu sen cambiando la conxugación del verbu quauxilia al infinitivu pol correllatu correspondiente: tuviera que quemalu únicamente amuesa'l conteníu obligativu que-y ye propiu a la perífrasis tener (quelde) + infinitivu.

L'exemplu 2 del infinitivu compuestu, debiin d'haber pisáolu, preséntase col complexu perifrásticu deber $(d e)+$ infinitivu + participiu. Nél esprésase la modalidá probabilística, la conxetura o la incertidume de qu'en dalgún momentu del pasáu asocediere l'aición de pisar, deduciéndose'l motivu pol que'l cristal ta rotu. Nesti contestu probabilísticu paez oponese a la forma con infinitivu simple debien pisalu en razón del valor terminativu de la forma compuesta: el refuerzu terminativu de l'aición paez nun se sorrayar esplícitamente na forma simple.

Con eso y con too, esti tipu de perífrasis haber + participiu aporta en determinaos contestos daqué carga terminativa que pue entrar en conflictu, en primeres, con tiempos eminentemente imperfeutivos. El pretéritu indefiníu de potencial -perfeutivu-debieron pisalu paez concasar col usu que se-y da a la forma compuesta debien d'haber pisáolu y nun entrar en conflictu col imperfeutu debien pisalu. La neutralización aspeutual d'estos tiempos verbales nun-y resulta estraña al asturianu (Viejo Fernández 1998: I8-22), polo qu'esta igualación nun se debe tanto al aspeutu terminativu y puen usase indistintamente.

Tampoco nun paez ser que la diferencia ente compuestu y forma simple cola perífrasis seya relevante como pa propiciar una oposición semántica funcional. Más entá, si tenemos en cuenta que’l condicional ye una forma más común y de munchu más rendimientu na fala pa 
espresar conteníos hipotético-probabilísticos en retrospeutiva. Por eso, qu'esti campu tien munches variables espresives:

Tien el cristal rotu.

Pisarienlu o algo. Pero anduvo mui bien. Nun sé si anda o no.

L'exemplu 4 d'infinitivu compuestu ye una oración truncada; polo tanto, presenta una serie de dificultaes d'interpretación. Intúise que ye un infinitivu compuestu que diría siguíu de participiu: Eso ye culpa mía de no haber [estudiao l'estándar llingüisticu] (vei muestra 16). Siguiendo esta conxetura, la utilización de la preposición de -pa introducir la oración subordinada sustantiva de complementu circunstancial de causaquiciabes se deba a un usu dialeutal que sustituya la preposición causal por con de. El castellán tampoco nun conoz de de la mesma manera, inclinándose l'usu tamién a la preposición por. Si s'utilizare de darrera del suxuntivu introduciría una oración condicional prospeutiva (Sedría culpa mio de nun estudiar l'estándar). Ye razonable consideralo una infracción carauterística de la llingua oral.

N'asturianu podría utilizase la forma correspondiente d'infinitivu simple (Eso ye culpa mía por nun estudiar l'estándar) ensin qu' eso supunxere alteración significativa qu'afectare al potencial espresivu del sistema verbal asturianu.

Con esta segunda riestra d'exemplos, pue apreciase la dificultá pa sustituyir l'infinitivu compuestu por una forma simple cuando tien d'auxiliar un verbu hipotético-probabilísticu nun planu inactual (tener $q u e+$ infinitivu + participiu). De facelo, piérdense determinaos conteníos: nos exemplos I, 3 y 5 la carga desiderativa. Esti fechu sofitaría aquelles hipótesis que defenden un probable desenrueldu autóctonu, pero non como un infinitivu compuestu definíu por oposición al simple, sinón funcionales a un nivel de perífrasis modal n'otros niveles del sistema (Viejo Fernández 1998: 53). Nun ye estensible a otros casos como’l 
del exemplu 2, onde l'auxiliar ye una perífrasis probabilística plena que nun da pie a solapaciones semántiques.

Per otru sitiu, nel exemplu 4 hai una interrupción na elocución, polo que vuelve a aprucir un indiciu importante de que les formes compuestes con haber puen considerase como marcadores llingüísticos del castellán. Na fala, a la escontra de la llingua escrita, la planificación y realización del discursu ye simultánea y por eso hai munchos contestos erráticos, pero la dulda del informante apaez nel mesmu momentu que'l de la muestra 13 -cuando la oración requería un participiu- independientemente de que se trate d'un usu propiu o impropiu del asturianu. L'informante d'Illas y la informante d'Uviéu reproducen el mesmu patrón.

\subsection{Exemplos de tener + participiu}

Les perífrasis tener + participiu apaecen en 5 muestres orales en sitios estremaos, conseñándose n'Uviéu y n'El Ferriru. Toes estes muestres coinciden na inmovilización del participiu en neutru perdiendo tola autonomía y col auxiliar tener deslexicalizáu nun presentando los valores propios del mesmu.

I. Alguna vez tien traiote y téngote sacao yo por abi pol Campillín en una silla.

2. Ye primero pa ella que pa vosotros, porque vino primero y tiénmelo dicho.

3. No lu tengo tratao, tratáu, sí, ye como tu na Colonia.

4. Aqui tiénenme buscao palanques de yerba a Tresona.

5. Si, d'eso tengo oío cuntar.

Munches construcciones comunes n'asturianu son desconocíes p'abondes de les variantes dialeutales del castellán; tenemos l'exemplu de la construcción tener + participiu (inmovilizáu en neutru) siguida d'infinitivu (cuntar). Los conteníos iterativos cuando en castellán utilicen tener, ye posible albidralos con participios de verbos transitivos, 
concordando en xéneru y númberu con CD: Tiene asegurada la plaza, tiene aseguradas las plazas, pero *tiene asegurado la plaza. N'asturianu puen construyise tanto con verbos transitivos como intransitivos, asina como con inmovilización sistemática del participiu en xéneru neutru (o masculín naquelles zones onde nun se dea). De toes maneres, l'usu pue amosar esa iteración como ye posible coles construcciones con haber + participiu, inferío por contestu: Según tengo oido, equivalente a Según he oido.

Un casu interesante ye'l del exemplu 3, qu’opón la perífrasis inmovilizada en neutru cola construcción que concuerda’l participiu y el CD (no lu tengo tratao, tratáu, sí [lu tengo]). La informante utiliza la construcción perifrástica nun lu tengo tratao con valor iterativu pero de pasáu (hasta agora nun lu traté muncho); por oposición, la variante con concordancia ente participiu sí lu tengo tratáu refierse a la esistencia de tratu presente. Una posible representación en castellán podría ser: No lo he tratado mucho hasta ahora, pero trato si que tengo.

Como estes construcciones son bastante xenerales dientro del asturianu y autóctones dafechu, nun ha estrañar que seyan les más regulares tanto na forma como nel significáu. En tolos casos tien un conteníu iterativu y nun presenta nengún problema pragmadiscursivu o morfosemánticu desconocíu.

\section{Conclusiones Xenerales}

Tres de tolo visto hasta agora, l'análisis de les muestres recoyíes amuesa qu'esiste un usu preferencial pa coles formes simples del verbu. Pue afirmase esto al contar con un corpus oral de más de diez hores de fala cotidiana con informantes repartíos per puntos estremaos del centru d'Asturies, onde namás se conseñen trece casos d'haber + participiu -muestres de Güeñu aparte- y seis con tener + participiu. 
Al usu reducíu d'esi tipu de formes compuestes hai que-y sumar la so atomización xeoleutal, centrándose cuasi esclusivamente n’Uviéu, zona urbana y con más contautu interllingüísticu (desequilibráu) asturianucastellán. Esti factor, pela cueta, nun afirma qu'haya suponése-yos un orixe castellán; nun ha suponése-yos en términos estrictos, porque, si bien la presión del castellán pue producir una xuba na incidencia d'eses formes por imitación -ya hibridación- cola fala de prestixu castellana, na codificación morfosemántica observóse una importante diferencia pragmadiscursiva: nos más de los casos les equivalencies son ayenes a los usos conocíos pol castellán.

Vense darréu los usos recoyíos nesta investigación nos distintos tipos de compuestos verbales:

Haber + participiu:

I) Aporta un reforzamientu terminativu o d'anterioridá en construcciones condicionales irreales en posición protática. Nun s'aprecia na forma simple nin na forma compuesta cuando va na apódosis (muestres I y 9). Neutraliza los valores d'equivalencia condicional-pretéritu pluscuamperfeutu del castellán en contestos sintáuticos d'oraciones condicionales d'irrealidá.

2) Espresión desiderativa y esclamativa con refuerzu terminativu. Infierse namás si consideramos que l'exemplu de la muestra 9 nun ye una condicional truncada, pero abárcales igualmente: Si lo hubiese echao fuera (;si lo hubiese echao fuera!), equivalente a ji lo echare fuera!

3) Neutralízase la distinción de pluscuamperfeutos n'indicativu y suxuntivu (muestres 7, 8 y II). Hubiera + participiu -pluscuamperfeutu de suxuntivu castellan- neutralízase colos valores pluscuamperfeutos d'asturianu (taparen) y castellán ( habian + participiu).

4) Claru enclín a apaecer en contestos sintáuticos subordinaos evidenciales introducíos por verbos parentéticos. Paez recurrente nes muestres 7,8 , II y I3. 
5) Con valor probabilísticu propiu de les perífrasis d'infinitivu compuestu con auxiliares hipotético-desiderativos (muestres de Güeñu). Tienen matices modales probabilísticos y non terminativos. Puen dir en posición absoluta (jhubiere muerto! = ¡Podía haber muerto!) como cláusula oracional independiente (muestra I de Güeñu).

Infinitivu compuestu haber + participiu:

I) Conteníu desiderativo-probabilísticu que nun tresmite la forma simple cuando ta auxiliáu por un verbu hipotético-desiderativu (poder o tener) nun planu inautual (muestres 2, 5 y Io). ;Tenía qu'haber + participiu! allúgase nuna perspeutiva retrospeutiva, de calter desiderativu y planu inautual. Nun comparte esos valores la forma simple, yá que produz un cambéu de perspeutiva (paralelo-prospeutiva) y perda del valor desiderativu.

Tener + participiu:

I) Espresa la retrospeutiva verbal secundaria con valores puramente iterativo-resultativos cuando'l participiu s'inmoviliza en neutru con verbos transitivos ya intransitivos indistintamente.

2) Focalización del valor aspeutual iterativu cuando'l participiu ta inmovilizáu en neutru frente al masculín (muestra 6). La falante siempre ye la referencia p'afitar la rellación temporal ente la perífrasis inmovilizada en neutru y la construcción de participiu concertáu.

La mayoría de les muestres de los compuestos haber + participiu cumplen un mesmu patrón: danse n'oraciones subordinaes y acompáñense de verbos parentéticos (saber que) en contestos nos que se marca la fonte d'información. Abonden dafechu en contestos claramente modales, seya dende oraciones condicionales -probabilístiques-, hipotéticoprobabilístiques, desideratives o rellacionaes colos contestos psicolóxicos d'evidencialidá. Los exemplos de Güeñu englóbense tamién nos contestos hipotético-probabilísticos, onde lo innovador ye la so apaición como cláusula independiente. 
Nes muestres con tener + participiu compruébase la inmovilización del participiu en neutru tanto n'Uviéu como n’El Ferriru. Amuesa más estabilidá morfosemántica que los complexos con haber + participiu. Esta invariabilidá caltiénse inalterada n'ámbitos estremaos como Uviéu y El Ferriru, anque nun faen oposición coles formes verbales simples, porque temporalmente son redundantes. Pero lo que sí ye plenamente funcional ye'l so valor iterativo-resultativu.

De cierto, son carauterizaciones poco frecuentes si atendemos a los datos, anque, al mesmu tiempu, d'un rendimientu evidente al so nivel na fala. Situación tala pue debese, en parte, a una promoción favorecida pola equiparación de construcciones asemeyaes a les de la llingua de prestixu, y non tanto a calcos semánticos por interferencia estructural, si bien tampoco hai que negar que puedan dase nuna situación de billingüismu socialmente desequilibráu; les interferencies a nivel léxicu son más qu'evidentes. Por embargu, ye un fechu que se constaten históricamente estos compuestos en documentaciones de la Edá Media y Moderna (Viejo Fernández I999: 42-43, 50-5I) con especial enfotu nos conteníos del pluscuamperfeutu ya imperfeutu de suxuntivu -ya infinitivu compuestu-, nos que, amás, tamién son escasos.

Nun ye fácil atopar contestos claros atribuyibles a castellanismu funcional. Más bien al revés, polo que la so esistencia seique tenga que ver con retazos vestixales de formes llatentes, pero que nun se xeneralizaren como nel restu de la Romania. Nun ámbitu peninsular, gallegu y asturianu son los únicos sistemes llingüísticos que nun xeneralizaron los compuestos, pero tampoco los desconocen. Amás, la situación xeográfica del asturianu sitúalu nuna zona intermedia, ente un sistema de tiempos compuestos funcionales (castellán) y otru de tiempos verbales simples (gallegu). A otru nivel, xustificaría la incidencia estremada nos diferentes ámbitos diatópicos del sistema asturianu. 
En síntesis, como rempuesta a los oxetivos marcaos pa esta investigación y tres del análisis de los datos recoyíos, deduzse como conclusión xeneral la constatación empírica de:

a) Mínima vitalidá, tanto a nivel d'asturianu como a nivel d'asturianu mínimu urbanu (AMU), con funcionalidá opositiva de los compuestos con haber + participiu llendada a contestos de pluscuamperfeutu ( hubiese/habia + participiu) asina como estructures morfosintáutiques subordinantes y d'evidencialidá.

b) El sistema verbal asturianu ye poco receutivu a la hora d'almitir formes compuestes castellanes; la cantidá mínima que se conseñó respuende a patrones pragmadiscursivos en clave asturiana. Apaecen en contestos modales con especializaciones que puen afondar nun desendolcu vestixal intrínsecu al sistema llingüísticu asturianu.

c) Les formes compuestes tener + participiu reproducen valores de marcáu calter iterativu que, en nengún casu, puen entendese como oposiciones análogues de los tiempos compuestos del castellán frente a les formes verbales simples.

Con eses, arriendes de tolo visto, albídrase tamién la fuerte carauterización como marcador llingüísticu del haber + participiu: considérase conscientemente como un elementu esternu; poro, non deseable nel discursu y que los propios falantes refuguen bramente (muestres I3 y I6). Nel grupu d'Uviéu y nel grupu d'Illas dase talamente la mesma situación cuando xurde la forma haber y precisa de participiu. Esto induz a pensar que ye dalgo xeneralizao nel ámbitu asturianu, pero tamién que los falantes puen ser más o menos conscientes d'usos foranos de les formes verbales cuando nun s'axusten a esquemes propiamente asturianos. 


\section{BIBLIOGRAFÍA}

Alarcos (i980) = Emilio Alarcos Llorach, «Perfecto simple y compuesto», en Estudios de gramática funcional del español, Madrid (Gredos), 1980, páxs. 13-49.

Alarcos (1987) = Emilio Alarcos Llorach, Estudios de gramática funcional del español, Madrid (Gredos), 1987.

ALLA (20OI) = ACADEMIA DE LA Llingua ASTURIANA, Gramática de la llingua asturiana ( $3 .^{\text {a }}$ edición), Uviéu (Academia de la Llingua Asturiana), 200 .

ALLA (2002) = ACADEMIA DE LA LLINGUA ASTURIANA, Informe sobre la llingua asturiana, ( $3 \cdot{ }^{a}$ edición), Uviéu (Academia de la Llingua Asturiana), 2002.

ANDRÉs (I993) = RAMÓN D'ANDRÉs, Encuesta sociollingüística nuna parroquia asturiana (Deva-Xixón), Uviéu (Universidá d'Uviéu), 1993.

ANDRÉs (I996) = RAMÓN D’ANDRÉs, «Encuestas sociolingüísticas sobre el asturiano: las variables de edad y sexo", Revista de Filología Románica, I3 (1996), páxs. 37I-388.

ANDRÉs (2002) = RAMÓN D’ANDRÉs, «L'asturianu mínimu urbanu: delles hipótesis», Lletres Asturianes, 8I (2002), páxs. 2I-38.

Bobes (I96I) = María del Carmen Bobes Naves, "Fonología del verbo bable», Boletín del Instituto de Estudios Asturianos, 42 (I96I), páxs. I03-II6.

Cano González (2009) = Ana María Cano González, El habla de Somiedo (Occidente de Asturias) (2. ${ }^{\mathrm{a}}$ ed.), Uviéu (Academia de la Llingua Asturiana), 2009 (I. ${ }^{\mathrm{a}}$ ed. I98I).

Cano González et alii (1976) = Ana María Cano González; María Victoria Conde Saiz; Xosé Lluis García Arias \& Francisco García González, Gramática bable, Uviéu (Naranco), I976.

Carrasco (2008) = Á. Carrasco Gutiérrez, Tiempos compuestos y formas verbales complejas, Madrid (Iberoamericana-Veuvert), 2008.

Cartagena (1999) = N. Cartagena, «Los tiempos compuestos del modo indicativo», en GDLE, vol. II, páxs. 2935-2975.

Catalán (1989) = Diego Catalán Menéndez-Pidal, Las lenguas circunvecinas del castellano. Cuestiones de dialectología hispano-románica, Madrid (Ed. Paraninfo), 1989, páxs. IOO-I3O.

Coseriu (I996) = Eugenio Coseriu, El sistema verbal románico, México (Siglo XXI Editores), 1996. 
Díaz Castañón (i966) = María del Carmen Díaz Castañón, El bable del Cabo Peñas. Contribución al estudio del bable central, Oviedo (Instituto de Estudios Asturianos), 1966.

Fernández de Castro (1990) = Félix Fernández de Castro, Las perifrasis verbales en español. Comportamiento sintáctico e historia de su caracterización, Oviedo (Universidad de Oviedo / Departamento de Filología), ı990.

Fernández de Castro (I999) = Félix Fernández de Castro, Las perifrasis verbales en el español actual, Madrid (Gredos), 1999.

Fernández Pérez (I993) = M. Fernández PÉrez, Las categorías gramaticas (morfológicas) en español, anexo 35 de Verba, Santiago de Compostela (Universidade de Santiago de Compostela), i993.

García Arias (1974) = José LuIs García Arias, XEl habla de Teberga: sincronía y diacronía, anexo XXIV de Archivum, Oviedo (Universidá d'Uviéu), I974.

GDLE = Ignacio Bosque Muñoz \& Violeta Demonte, Gramática descriptiva de la lengua española, Madrid (Espasa Calpe / Real Academia Española), I999.

Hurtado (I998) = S. Hurtado González, «El perfecto simple y el perfecto compuesto en el español actual: estado de la cuestión», Epos, XIV (I998), páxs. 5I-67.

Lázaro Carreter (1984) = Fernando Lázaro Carreter, Diccionario de términos filológicos, Madrid (Gredos), 1984.

Llano Caelles (I993) = Xilberto Llano Caelles, "Apuntes del paradigma verbal asturianu», Lletres Asturianes, 46 (I993), páxs. 49-59.

Martínez Álvarez (I973) = Josefina Martínez Álvarez, «Las formas compuestas en el verbo del bable central», Archivum, XXIII (1973), páxs. 299-307.

Muñız (1976) = Celso MuñIz, El habla del Valledor. Estudio descriptivo del gallegoasturiano de Allande (Asturias-España), Amsterdam (Academische Pers), 1976.

Neira (I976) = Jesús Neira Martínez, El bable: estructura e historia, Salinas (Ayalga), I976.

NGLE = Real Academia Española, Nueva gramática de la lengua española, Madrid (Espasa Libros), 20Io.

Reyes Montero (20I4) = J. F. Reyes Montero, «Tiempo y aspecto en español y en las lenguas románicas» (trabajo fin de grado, Universidad de Cádiz, 20I4).

Rodríguez-Castellano (1954) = Lorenzo Rodríguez-Castellano, Aspectos del bable occidental, Oviedo (Instituto de Estudios Asturianos), I954. 
Rojo (I990) = GuILlermo Rojo SÁnCHEZ, «Relaciones entre temporalidad y aspecto en el verbo español», en Ignacio Bosque (ed.), Tiempo y aspecto en español, Madrid (Cátedra), I990, páxs. 17-43.

Teso (2015) = Enrique del Teso Martín, «L'amestáu urbanu. Cuestiones de normativa y llingua escrita», Lletres Asturianes, II3 (20I5), páxs. II-26.

Tobón \& Rodríguez (1974) = L. Tobón de Castro \& J. Rodríguez Rondón, «Algunas consideraciones sobre el aspecto verbal en español», Thesaurus. Boletín del Instituto Caro y Cuervo, 29/I (1974), páxs. 34-47.

Viejo Fernández (I998) = Xulio Viejo Fernández, Las formas compuestas en el sistema verbal asturiano, Oviedo (Universidad de Oviedo / Departamento de Filología Española), I998.

Viejo Fernández (I999) = Xulio Viejo Fernández, «Les formes compuestes del verbu asturianu nel contestu hispánicu: estudiu diacrónicu y comparativu", Lletres Asturianes, 69 (1999), páxs. 27-60.

Viejo Fernández (200I) = Xulio Viejo Fernández, «Algunos apuntes pragmáticos sobre el continuo asturiano", Archivum, 48-49 (200I), páxs. 54I-572.

Viejo Fernández (20II/2OI2) = Xulio Viejo FernándeZ, «Pasiva, parámetros determinativos y proyección discursiva n'asturianu oral», Archivum, 6I-62 (2OII-I2), páxs. 53I-560. 

